\title{
Short communication: Genetic relationships between functional longevity and direct health traits in Austrian Fleckvieh cattle
}

\author{
C. Pfeiffer, ${ }^{* 1}$ C. Fuerst, $†$ V. Ducrocq, $\ddagger$ and B. Fuerst-Walt|* \\ *University of Natural Resources and Life Sciences (BOKU), Department of Sustainable Agricultural Systems, Division of Livestock Sciences, \\ Vienna, Gregor-Mendel-Straße 33, A-1180 Vienna, Austria \\ †ZuchtData EDV-Dienstleistungen GmbH, Dresdner Straße 89/19, A-1200 Vienna, Austria \\ ‡INRA, UMR 1313, Génétique Animale et Biologie Intégrative (GABI), 78350 Jouy-en-Josas, France
}

\begin{abstract}
The aim of this study was to conduct a multitrait 2-step approach applied to yield deviations and deregressed breeding values to get genetic parameters of functional longevity, clinical mastitis, early fertility disorders, cystic ovaries, and milk fever of Austrian Fleckvieh cattle. An approximate multitrait approach allows the combination of information from pseudophenotypes derived from different statistical models in routine genetic evaluation, which cannot be estimated easily in a full multitrait model. A total of 66,890 Fleckvieh cows were included in this study. For estimating genetic parameters, a simple linear animal model with year of birth as a fixed effect and animal as a random genetic effect was fitted. The joint analysis of yield deviations and deregressed breeding values was feasible. As expected, heritabilities were low, ranging from 0.03 (early fertility disorders) to 0.15 (functional longevity). Genetic correlations between functional longevity and clinical mastitis, early fertility disorders, cystic ovaries, and milk fever were $0.63,0.29,0.20$, and 0.20 , respectively. Within direct health traits genetic correlations were between 0.14 and 0.45 . Results suggest that selecting for more robust disease-resistant cows would imply an improvement of functional longevity.
\end{abstract}

Key words: approximate multiple trait, health trait, functional longevity, genetic parameter, cattle

\section{Short Communication}

The importance of functional traits in modern dairy breeding programs is increasing worldwide. Besides a broad range of functional traits such as fertility, longevity, and calving traits, direct health traits gained more importance due to their effects on farm economy, animal welfare, and customers' concerns about food safety recently (Egger-Danner et al., 2012). Since 2010,

Received March 26, 2015.

Accepted July 2, 2015

${ }^{1}$ Corresponding author: christina.pfeiffer@boku.ac.at clinical mastitis (CM), early fertility disorders (EFD), cystic ovaries (CO), and milk fever (MF) are an integral part of the routine genetic evaluation of Austrian Fleckvieh cattle (dual-purpose Simmental; Fuerst et al., 2011). Currently, these traits are evaluated separately. As reported for other populations (Ducrocq et al., 2001), true genetic and residual correlations or heterogeneous reliabilities are neglected when these traits are subsequently combined into a total merit index or other subindices. Functional traits usually have a low additive genetic variance and are antagonistically correlated with production traits. However, lowly heritable traits benefit from being analyzed together with highly heritable correlated traits in a multivariate approach. Accuracy can be increased compared with univariate analysis due to simultaneous genetic evaluation of correlated traits and better data connectedness because genetic and residual covariances between traits are included. A full multivariate estimation of all traits based on phenotypic data could be considered as the optimum methodology, but is usually not feasible (Mrode, 2014) due to the large numbers of traits involved in a breeding program. Therefore, an approximate multitrait approach was proposed by Ducrocq et al. (2001) and validated on simulated data by Lassen et al. (2007) and Pfeiffer et al. (2015). Apart from an increase of accuracy, Lassen et al. (2007) could show that using an approximate multitrait model for predicting breeding values led to a higher genetic trend compared with univariate analysis. Estimated breeding values were not significantly different when computed in an approximate multitrait approach compared with a full multitrait model based on phenotypic data (Lassen et al., 2007; Pfeiffer et al., 2015). This genetic trend is unbiased by selection on one or several correlated traits. Finally, these EBV can be easily combined in a total merit index (TMI) because the optimum weights in the TMI are simply the trait economic values when a multiple trait evaluation is performed. In Austrian Fleckvieh cattle, the main reasons for culling are fertility disorders (22.9\%) and udder diseases (13.5\%; ZuchtData, 2015). Disease-related losses are high in 
dairy cattle production (Beaudeau et al., 1999). Health disorders lead to early culling and decrease longevity. Several estimates of heritabilities of health traits and a few estimates of genetic correlations between them are reported in literature (e.g., Heringstad et al., 2005; Koeck et al., 2010; Fuerst et al., 2011). However, genetic correlations between health traits and functional longevity are not available due to no or limited access to direct health data in most countries (Egger-Danner et al., 2012) and due to methodology constraints. Hence, the objectives of this study were to conduct an approximate multitrait 2-step approach applied to yield deviations (YD) and deregressed breeding values (drEBV) deducted from different statistical models and to estimate heritabilities, genetic and residual correlations between functional longevity (LONG), CM, EFD, CO, and MF in Austrian Fleckvieh cattle. In total, 66,890 Fleckvieh cows of 2 Austrian regions, with a maximum of $12.5 \%$ non-Fleckvieh-gene proportion, recorded for all 5 traits and born between 2004 and 2009, were selected for estimating genetic parameters. Data were further restricted to sires, which were progeny tested and had at least 20 daughters, whereas only the first 1,000 daughters were considered for highly used sires. Their pedigree was traced back as far as possible, yielding 202,430 animals. All health traits were recorded as binary traits $(0=$ healthy, $1=$ diseased within a certain time period) in all lactations. As described in Fuerst et al. (2011), CM consists of acute and chronic mastitis $10 \mathrm{~d}$ ante-partum (a.p.) to $150 \mathrm{~d}$ postpartum (p.p.). Diagnosis of retained placenta, puerperal diseases, or metritis from day of calving until $30 \mathrm{~d}$ p.p. was recorded as EFD. The observation period for CO was $30 \mathrm{~d}$ p.p. to $150 \mathrm{~d}$ p.p. The MF was recorded $10 \mathrm{~d}$ a.p. to $10 \mathrm{~d}$ p.p. If cows were involuntarily culled within the described time spans and because of udder health problems, fertility disorders, or metabolic diseases, their respective diagnosis was also recorded. These animals were considered as diagnosed in the health monitoring system and were also included in the analysis. In the first step, longevity was analyzed using the Survival Kit v6 software (Mészáros et al., 2013). The following statistical model is currently used in the joint routine genetic evaluation of Austria and Germany:

$$
\begin{gathered}
\mathrm{h}(\mathrm{t})=\mathrm{h}_{0, \mathrm{ls}}(\tau) \times \exp \left\{\Sigma \mathrm { m } \left[\mathrm{f}_{\mathrm{m}}(\mathrm{t})+\mathrm{hy}_{\mathrm{k}}(\mathrm{t})\right.\right. \\
\left.\left.+\mathrm{s}_{\mathrm{i}}+0.5 \mathrm{mgs}_{\mathrm{j}}\right]\right\}
\end{gathered}
$$

where $\mathrm{h}(\mathrm{t})$ is the hazard of a cow $\mathrm{t}$ days after her first calving; $h_{0, l s}(\tau)$ is the Weibull baseline hazard function per lactation $l$ and stage of lactation $\mathrm{s}$ with scale parameter $\lambda$ and shape parameter $\rho ; \mathrm{hy}_{\mathrm{k}}(\mathrm{t})$ is the random time dependent effect of herd-year following a log- gamma distribution; $f_{m}(t)$ represent the fixed effects of region-year-season, age at first calving, relative performance within herd (fat + protein yield; time dependent), change of herd size (time dependent), an indicator of alpine pasturing (time dependent), and heterosis and recombination loss; $s_{i}$ is the random sire genetic effect; and $\mathrm{mgs}_{\mathrm{j}}$ is the random maternal grandsire genetic effect. The random effects $s_{i}$ and $\mathrm{mgs}_{j}$ were assumed to follow a multivariate normal distribution with mean zero and variance $\mathbf{A} \sigma_{s}^{2}$, where $\sigma_{s}^{2}$ is the variance among sires and $\mathbf{A}$ is the relationship matrix. The YD equivalents and their corresponding weights were computed as a function of the cumulative hazard of each particular individual [for a more detailed description, see Ducrocq (2001) and Ducrocq et al. (2001)].

Breeding values for health traits were estimated univariately using following linear animal model:

$$
\mathbf{y}=\mathbf{X b}+\mathbf{Z}_{\mathbf{h}} \mathbf{h}+\mathbf{Z}_{\mathrm{a}} \mathbf{a}+\mathbf{W} \mathbf{p}+\mathbf{e},
$$

where $\mathbf{y}$ is the vector of observations of $\mathrm{CM}, \mathrm{EFD}, \mathrm{CO}$, and $\mathrm{MF} ; \mathbf{b}$ is the vector of systematic effects, including fixed effects of parity $\times$ age at calving, calving year and month, and type of recording (electronically by the veterinarians or by the performance recording organization during routine milk recording) by year; $\mathbf{h}$ is the vector of random herd-year effects with $\mathrm{N}\left(0, \mathbf{I} \sigma_{\mathrm{h}}^{2}\right)$, where $\mathbf{I}$ is the identity matrix and $\sigma_{\mathrm{h}}^{2}$ represents the herd-year variance; $\mathbf{a}$ is the vector of random additive genetic effects with $N\left(0, \mathbf{A} \sigma_{\mathrm{a}}^{2}\right)$, where $\mathbf{A}$ represents the numerator relationship matrix and $\sigma_{\mathrm{a}}^{2}$ represents the animal variance; $\mathbf{p}$ is the vector of the permanent environmental effects of the cow; $\mathbf{e}$ is the vector of the random residual effects, with $N\left(0, \mathbf{I} \sigma_{\mathrm{e}}^{2}\right)$, where $\mathbf{I}$ is the identity matrix and $\sigma_{\mathrm{e}}^{2}$ is the residual variance. The $\mathbf{X}$, $\mathbf{Z}_{\mathrm{h}}, \mathbf{Z}_{\mathrm{a}}$, and $\mathbf{W}$ represent the corresponding incidence matrices (Fuerst et al., 2011). Health traits were then deregressed using a univariate de-regression based on the approach of Jairath et al. (1998) and Schaeffer (2001), which is implemented in the program package MiX99 (Lidauer et al., 2013). The de-regression procedure uses the estimated breeding values and their respective effective daughter contributions as weights only considering the general mean as fixed effect. Based on approximate Interbull reliabilities (Strandén et al., 2000), effective own performances (Edel et al., 2009) were calculated and used as weighting factors for drE$\mathrm{BV}$ in the multivariate estimation of genetic parameters. In the second step, after computing YD equivalent for LONG and drEBV for CM, EFD, CO, and MF, respectively, a multivariate animal model was fitted applying an average information algorithm using the AS- 
Reml package (Gilmour et al., 2009). The following model was used:

$$
\mathbf{y}^{*}=\mathbf{X b}+\mathbf{Z a}+\mathbf{e}
$$

where $\mathbf{y}^{*}$ indicates the YD for LONG and drEBV for $\mathrm{CM}, \mathrm{EFD}, \mathrm{CO}$, and $\mathrm{MF}$ for a given animal, respectively; $\mathbf{X}$ and $\mathbf{Z}$ represent the incidence matrices for fixed and random effects, respectively; $\mathbf{b}$ is the vector of the fixed year of birth effects; $\mathbf{a}$ is the vector of the random additive genetic effects; and $\mathbf{e}$ is the vector of the random residual effects. Vector a was assumed to have multivariate normal distribution, with $\operatorname{MVN}(0$, $\mathbf{G}=\mathbf{G}_{0} \otimes \mathbf{A}$ ), where $\mathbf{G}_{0}$ is a $5 \times 5$ additive genetic variance-covariance matrix, $\otimes$ is the Kronecker product of matrices, and $\mathbf{A}$ represents the numerator relationship matrix. Residuals e were assumed to be $\operatorname{MVN}(0$, $\mathbf{R}=\mathbf{R}_{0} \otimes \mathbf{I}$ ), where $\mathbf{R}_{0}$ is a $5 \times 5$ residual variancecovariance matrix and $\mathbf{I}$ represents the identity matrix. In the first step of the analysis, the computation of pseudo-records was performed in univariate analyses ignoring correlations between traits even if this might lead to a possible bias in genetic trends. In the second step, a multivariate model was applied. To correct for a potential selection bias, a year effect was included (Lassen et al., 2007). Based on estimated (co)variance components, heritabilities, genetic and residual correlations were calculated.

Results of this study showed that the joint analysis of longevity YD equivalent and drEBV in an approximate 2-step approach was feasible. Our 2-step approach had already been validated on simulated data (Pfeiffer et al., 2015). Results of the simulation study showed almost no bias of YD and drEBV compared with a full multivariate model based on phenotypic data. Furthermore, estimates of genetic correlations between health traits were in accordance with results presented in literature (e.g., Heringstad et al., 2005). Although the applied model is simple, solving large mixed model equations is still a challenge in terms of computing power (Lassen et al., 2007). Computation of the 5 trait animal model was undertaken on an Intel Xeon CPU E5520 at $2.27 \mathrm{GHz}$ with 75 GB of RAM. Convergence of the AI-REML algorithm was reached after 15 iterations in almost $40 \mathrm{~h}$.

Heritabilities, and genetic and residual correlations of all 5 traits are given in Table 1 (positive values are favorable genetic correlations). Heritabilities of all traits were in the range of heritabilities estimated for routine genetic evaluation (Fuerst et al., 2015). Nevertheless, they are not directly comparable with usual estimates obtained from phenotypic data. Due to the different number of records of each animal, residual (co)variances are (co)variances between averages of residuals. All genetic correlations, except the one between $\mathrm{CM}$ and EFD, were significantly different from zero as standard errors were relatively small (0.05 to 0.09). Residual correlations were almost zero. Estimates of genetic correlations $\left(\mathrm{r}_{\mathrm{a}}\right)$ between LONG and CM, EFD, $\mathrm{CO}$, and $\mathrm{MF}$ were moderate to high; the highest value was found between LONG and CM $\left(r_{a}=0.63\right)$. Cows that are more resistant to mastitis are hence less likely to be culled. Also based on an approximate 2-step approach, similar results were reported for different French dairy breeds (Govignon-Gion et al., 2012). The authors estimated slightly lower genetic correlations of $\mathrm{r}_{\mathrm{a}}=-0.47$ to -0.56 (in their case, negative values were favorable) between LONG and CM, applying an approximate 2-step approach. Neerhof et al. (2000) compared the risk for being culled between cows suffering from mastitis to their healthy herd cohorts and noted that the risk of being culled is 1.69 times greater for animals with mastitis. Genetic correlations between LONG and EFD, CO, and MF were 0.29, 0.20, and 0.20 , respectively. Selecting for more robust disease resistant cows entails an improvement of LONG due to a strong genetically favorable correlated response. Genetic correlations between health traits were low to moderate, ranging from 0.14 between $\mathrm{CM}$ and EFD to 0.45 between CO and MF. Similar results of genetic correlations between CM and several fertility disorders were found in earlier studies (Heringstad et al., 2005; Koeck et al., 2010, 2012). Genetic correlations between the metabolic disease $\mathrm{MF}$ and $\mathrm{CM}, \mathrm{EFD}$, and $\mathrm{CO}$ $(0.37,0.34$, and 0.45$)$, respectively, were higher than

Table 1. Heritabilities (on the diagonal), genetic correlations (above diagonal) and residual correlations (below diagonal) and their standard errors in parentheses for functional longevity (LONG), clinical mastitis (CM), early fertility disorders (EFD), cystic ovaries (CO), and milk fever $(\mathrm{MF})^{1}$

\begin{tabular}{lccccc}
\hline Trait & LONG & CM & EFD & CO & MF \\
\hline LONG & $0.15(0.01)$ & $0.63(0.05)$ & $0.29(0.08)$ & $0.20(0.07)$ & $0.20(0.08)$ \\
CM & $0.09(0.006)$ & $0.06(0.01)$ & $0.14(0.09)$ & $0.16(0.07)$ & $0.37(0.08)$ \\
EFD & $0.02(0.006)$ & $0.02(0.005)$ & $0.03(0.01)$ & $0.42(0.08)$ & $0.34(0.09)$ \\
CO & $-0.02(0.006)$ & $-0.01(0.005)$ & $0.02(0.005)$ & $0.07(0.01)$ & $0.45(0.07)$ \\
MF & $0.02(0.006)$ & $0.004(0.005)$ & $0.02(0.004)$ & $-0.004(0.005)$ & $0.05(0.01)$ \\
\hline
\end{tabular}

${ }^{1}$ Heritabilities and residual correlations are not directly comparable with estimates from phenotypic data. 
values from previous studies. Heringstad et al. (2005) estimated genetic correlations between $\mathrm{MF}$ and $\mathrm{CM}$ and retained placenta of 0.11 and 0.18 in third lactating cows. Cows that phenotypically suffer from MF have a higher risk to suffer from other homeostatic disorders such as CM, fertility disorders, or other metabolic diseases later (DeGaris and Lean, 2008). Although CM, EFD, CO, and MF can be considered as different traits, a partly common genetic background exists. Selecting against diseases that frequently occur and show moderate genetic correlations to other health traits such as $\mathrm{CM}$ or $\mathrm{MF}$ also increases the resistance to some other diseases. Thus, a general disease resistance factor with a genetic component might exist (Heringstad et al., 2005). Results of the current study confirm these findings. Therefore, a strict selection for healthy cows is highly recommended to increase longevity and consequently positively influence farm economics and animal welfare.

\section{ACKNOWLEDGMENTS}

The authors acknowledge the funding of the project OptiGene (Optimization of long-term genetic progress of Austrian cattle breeds with emphasis on health and genomic selection; project number 100808) by the Austrian Federal Ministry of Agriculture, Forestry, Environment and Water Management; the Federations of Austrian Fleckvieh (Zwettl, Austria), Brown Swiss (Innsbruck, Austria), Pinzgauer (Maishofen, Austria) and Tyrolean Grey cattle (Innsbruck, Austria); and the Federation of Austrian Cattle Breeders (ZAR, Vienna, Austria).

\section{REFERENCES}

Beaudeau, F., H. Seegers, V. Ducrocq, and C. Fourichon. 1999. Effect of health disorders on culling in dairy cows: A review and critical discussion. Interbull Bull. 21:139-151.

DeGaris, P. J., and I. J. Lean. 2008. Milk fever in dairy cows: A review of pathophysiology and control principles. Vet. J. 176:58-69.

Ducrocq, V. 2001. A two-step procedure to get animal model solutions in Weibull survival models used for genetic evaluations on length of productive life. Interbull Bull. 27:147-152.

Ducrocq, V., D. Boichard, A. Barbat, and H. Larroque. 2001. Implementation of an approximate multitrait BLUP evaluation to combine production traits and functional traits into a total merit index. Pages 7-14 in Proc. 52nd Annual Meeting EAAP, Budapest, Hungary. Wageningen Academic Publisher, Wageningen, the Netherlands.
Edel, C., R. Emmerling, and K. U. Götz. 2009. Optimized aggregation for MA-BLUP evaluation in German Fleckvieh. Interbull Bull. 40:178-183.

Egger-Danner, C., B. Fuerst-Waltl, W. Obritzhauser, C. Fuerst, H. Schwarzenbacher, B. Grassauer, M. Mayerhofer, and A. Koeck. 2012. Recording of direct health traits in Austria-Experience report with emphasis on aspects of availability for breeding purposes. J. Dairy Sci. 95:2765-2777.

Fuerst, C., J. Dodenhoff, C. Egger-Danner, R. Emmerling, H. Hamann, D. Krogmeier, and H. Schwarzenbacher. 2015. Zuchtwertschätzung beim Rind-Grundlagen, Methoden und Interpretation. Accessed Jun. 10, 2015. http://zar.at/Downloads/Zuchtwerte.html.

Fuerst, C., A. Koeck, C. Egger-Danner, and B. Fuerst-Waltl. 2011. Routine genetic evaluation for direct health traits in Austria and Germany. Interbull Bull. 44:210-215.

Gilmour, A. R., B. J. Gogel, B. R. Cullis, and R. Thompson. 2009. ASReml User Guide Release 3.0. VSN International Ltd., Hemel Hempstead, UK.

Govignon-Gion, A., R. Dassonneville, G. Balloche, and V. Ducrocq. 2012. Genetic evaluation of mastitis in dairy cattle in France. Interbull Bull. 46:121-126.

Heringstad, B., Y. M. Chang, D. Gianola, and G. Klemetsdal. 2005. Genetic analysis of clinical mastitis, milk fever, ketosis, and retained placenta in three lactations of Norwegian red cows. J. Dairy Sci. 88:3273-3281.

Jairath, L., J. C. M. Dekkers, L. Schaeffer, Z. Liu, E. B. Burnside, and B. Kolstad. 1998. Genetic evaluation for herd life in Canada. J. Dairy Sci. 81:550-562.

Koeck, A., C. Egger-Danner, C. Fuerst, W. Obritzhauser, and B. Fuerst-Waltl. 2010. Genetic analysis of reproductive disorders and their relationship to fertility and milk yield in Austrian Fleckvieh dual-purpose cows. J. Dairy Sci. 93:2185-2194.

Koeck, A., F. Miglior, D. F. Kelton, and F. S. Schenkel. 2012. Health recording in Canadian Holsteins: Data and genetic parameters. J. Dairy Sci. 95:4099-4108.

Lassen, J., M. K. Sørensen, P. Madsen, and V. Ducrocq. 2007. An approximate multitrait model for genetic evaluation in dairy cattle with robust estimation of genetic trends. Genet. Sel. Evol. 39:353367.

Lidauer, M., K. Matilainen, E. Mäntysaari, T. Pitkänen, M. Taskinen, and I. Strandén. 2013. MiX99. General program for solving large mixed model equations with preconditioned conjugate gradient method. Release VII/2013 beta, Jokioinen, Finland.

Mészáros, G., J. Sölkner, and V. Ducrocq. 2013. The Survival Kit: Software to analyze survival data including possibly correlated random effects. Comput. Methods Programs Biomed. 110:503-510.

Mrode, R. A. 2014. Linear Models for the Prediction of Animal Breeding Values, 3rd ed. CAB Int., Wallingford, UK.

Neerhof, H. J., P. Madsen, V. P. Ducrocq, A. R. Vollema, J. Jensen, and R. Korsgaard. 2000. Relationships between mastitis and functional longevity in Danish black and white dairy cattle estimated using survival analysis. J. Dairy Sci. 83:1064-1071.

Pfeiffer, C., B. Fuerst-Waltl, H. Schwarzenbacher, F. Steininger, and C. Fuerst. 2015. A comparison of methods to calculate a total merit using stochastic simulation study. Genet. Sel. Evol. 47:36.

Schaeffer, L. 2001. Multiple trait international bull comparison. Livest. Prod. Sci. 69:145-153.

Strandén, I., M. Lidauer, E. A. Mäntysaari, and J. Pösö. 2000. Calculation of Interbull weighting factors for the Finnish test day model. Interbull Bull. 26:78-79.

ZuchtData. 2015. ZuchtData Jahresbericht, 2014. ZuchtData EDVDienstleistungen GmbH, Vienna. Accessed Feb. 20, 2015. http:// zar.at/Downloads/Jahresberichte/ZuchtData-Jahresberichte.html. 